\title{
NANOPARTÍCULES LIPÍDIQUES D’UN FLAVONOIDE D'ORIGEN NATURAL PER AL TRACTAMENT DEL GLAUCOMA
}

\author{
ZANFIREsCU, VASIle Marian; ${ }^{1}$ Bonilla Vidal, Lorena; \\ Espina, Marta; Pujol Cubells, Montserrat; García, Maria Luisa; \\ SÁNCHEZ-LÓPEZ, ELENA \\ Secció departamental de Fisicoquímica \\ Departament de Farmàcia, Tecnologia Farmacèutica i Fisicoquímica \\ Facultat de Farmàcia i Ciències de l'Alimentació \\ Universitat de Barcelona
}

\begin{abstract}
Glaucoma is a chronic, gradual and very serious neurodegenerative disease, and its natural course is blindness. In fact, it is the leading cause of irreversible blindness in the world. Current treatments have several limitations, such as limited bioavailability and low protection of the optic nerve. Therefore, in this work the development of a formulation containing second-generation lipid nanoparticles, known as nanostructured lipid carriers (NLCs), loaded with a natural flavonoid poorly soluble in water has been undertaken. For the synthesis of these NLCs, the hot high-pressure homogenization method has been used, where a high amount of energy is applied. Using the optimized formulation, the HET-CAM test was performed, an in vitro ocular tolerance test on embryonic eggs, classifying the formulation as practically non-irritating.
\end{abstract}

Keywords: flavonoid, NLC (nanostructured lipid carriers), eye, HET-CAM test.

\section{Resumen}

El glaucoma es una enfermedad neurodegenerativa crónica, gradual, muy grave, y su curso natural es la ceguera. De hecho, es la principal causa de ceguera irreversible en el mundo. Los tratamientos actuales tienen una serie de limitaciones, como son la biodisponibilidad limitada y la baja protección del nervio óptico. Por tanto, en este trabajo se lleva a cabo el desarrollo de una formulación que contiene nanopartículas lipídicas de segunda generación, conocidas con el nombre de «transportadores lipídicos nanoestructurados» (de las siglas en inglés de nanostructured lipid carriers, NLC), cargadas con un flavonoide natural que es muy poco soluble en agua. Para la síntesis de estos NLC se ha utilizado el método de homogenización de alta presión en caliente, donde se aplica una elevada cantidad de energía. Utilizando la formulación optimizada se ha llevado a cabo la prueba HET-CAM, un ensayo de tolerancia ocular in vitro en huevos embrionados, clasificando la formulación como prácticamente no irritante.

Palabras clave: flavonoides, NLC (transportadores lipídicos nanoestructurados), ojo, test HET-CAM.

\section{Resum}

El glaucoma és una malaltia neurodegenerativa crònica, gradual, molt greu, i el seu curs natural és la ceguera. De fet, és la principal causa de ceguera irreversible al món. Els tractaments actuals tenen una sèrie de limitacions, com són la biodisponibilitat limitada i la baixa protecció del nervi òptic. Per tant, en aquest treball es porta a terme el desenvolupament d'una formulació que conté nanopartícules lipídiques de segona generació, conegudes amb el nom de

\footnotetext{
${ }^{1}$ Graduat en Farmàcia per la UB. Farmacèutic en oficina de Farmàcia (mariiaann28@hotmail.com).
} 
«transportadors lipídics nanoestructurats» (de les sigles en anglès de nanostructured lipid carriers, NLC), carregades d'un flavonoide natural que és molt poc soluble en aigua. Per a la síntesi d'aquests NLC s'ha utilitzat el mètode d'homogeneïtzació d'alta pressió en calent, on s'aplica una elevada quantitat d'energia. Fent servir la formulació optimitzada s'ha dut a terme el test HET-CAM, un assaig de tolerància ocular in vitro en ous embrionats, classificant la formulació com a pràcticament no irritant.

Paraules clau: flavonoides, NLC (transportadors lipídics nanoestructurats), ull, test HET-CAM.

\section{Introducció}

«Glaucoma» és un terme utilitzat per descriure un grup de trastorns que tenen en comú una degeneració progressiva del nervi òptic que causa dificultats visuals i eventualment ceguera. Col-lectivament, el glaucoma és la principal causa de ceguera irreversible al món. La pressió intraocular elevada és un factor de risc important per molts dels tipus de glaucoma (Wiggs i Pasquale, 2017). La majoria dels casos són asimptomàtics fins que la lesió del nervi òptic avança i es desenvolupa la pèrdua del camp visual. Podem classificar el glaucoma segons l'existència d'obstrucció al sistema de drenatge de l'humor aquós, com glaucoma d'angle obert o d'angle tancat. Quan el drenatge de l'humor aquós no és el correcte, en la cambra anterior de l'ull es produeix un augment de la pressió, que es transmet a la cambra posterior (humor vitri) i produeix una disminució del flux sanguini de la retina. Això, alhora, produeix una mala irrigació arterial de la papillla òptica que es tradueix en una degeneració progressiva de les fibres del nervi òptic i, per tant, en una ceguera progressiva (Lora i Andr, 2005).

El tractament del glaucoma depèn de la causa, dels factors de risc, de la gravetat i del tipus de glaucoma (Yadav, Rajpurohit i Sharma, 2019). Un dels factors de risc és l'elevada pressió intraocular de l'ull, de manera que ens interessa reduir aquesta pressió per aturar la progressió del curs de la malaltia. La reducció de la pressió intraocular es pot realitzar mitjançant diversos mètodes, és a dir, amb tractament làser, cirurgia i tractament farmacològic. Dins del tractament farmacològic s'inclouen fàrmacs de diverses classes, com ara anàlegs de les prostaglandines, $\beta$-blocadors, inhibidors de l'anhidrasa carbònica, agonistes adrenèrgics, miòtics i agents hiperosmòtics. Aquests fàrmacs o bé incrementen el drenatge del fluid de l'ull o bé disminueixen la producció d'aquest fluid (McAlinden, 2014). Malgrat això, el glaucoma mai no es pot curar completament.

El desenvolupament de la nanotecnologia ha canviat el panorama científic de la indústria sani- tària i ha donat lloc a noves aproximacions quant a medicaments i alliberament de fàrmacs. A més, aplicar la nanotecnologia en oftalmologia ha permès el desenvolupament de nous sistemes d'allibe- rament de fàrmacs, com les nanopartícules pel tractament de diverses malalties oculars. Aquestes nanopartícules, doncs, s'utilitzen com a vehicles per als fàrmacs per ser alliberats (Soni i Yadav, 2015). Dins de les nanopartícules biodegradables es troben les nanopartícules lipídiques i, concretament, els NLC, que proporcionen una sèrie d'avantatges, com l'increment de la biodisponibilitat i un alliberament perllongat del fàrmac. En aquest treball s'ha incorporat als NLC un flavonoide d'origen natural insoluble en un medi aquós. Aquest flavonoide natural presenta propietats neuro- protectores que podria alentir la progressió del curs de la malaltia (Youdim et al., 2002). Un dels principals efectes adversos de les formulacions oftàlmiques és la irritació ocular; per tant, per avaluar si la formulació és o no irritant a nivell ocular s'ha utilitzat una alternativa del test de Draize, que és el test HET-CAM, un assaig econòmic i sensible que utilitza ous de gallina embrionats (Gilleron et al., 1996). El test HET-CAM és un assaig establert per a irritacions oculars i ha demostrat una bona cor-relació amb el grau d'irritació ocular in vivo (Hagino et al., 1999; the hen's egg test-chorioallantoic membrane (HET-CAM Gupta et al., 2010), donat que la xarxa vascular funcional de la placenta de l'embrió del pollastre s'aproxima a la vasculatura ocular humana. Aquest assaig constitueix una tèc-nica in vitro, tot i que no substitueix els estudis in vivo, però ens serveix a priori per saber si una substància és potencialment irritant a nivell ocular o no. 


\section{Disseny}

\subsection{Marc teòric}

\subsubsection{Anatomia de l'ull}

Per comprendre el glaucoma, primer s'ha d'entendre quina és la funció de l'ull. La figura 1 presenta un esquema per identificar les parts més importants de l'ull. L'escleròtica és una capa blanca i resistent que serveix per protegir l'ull; part d'aquesta es pot observar davant de l'ull. La conjuntiva és una membrana transparent i delicada que cobreix l'escleròtica.

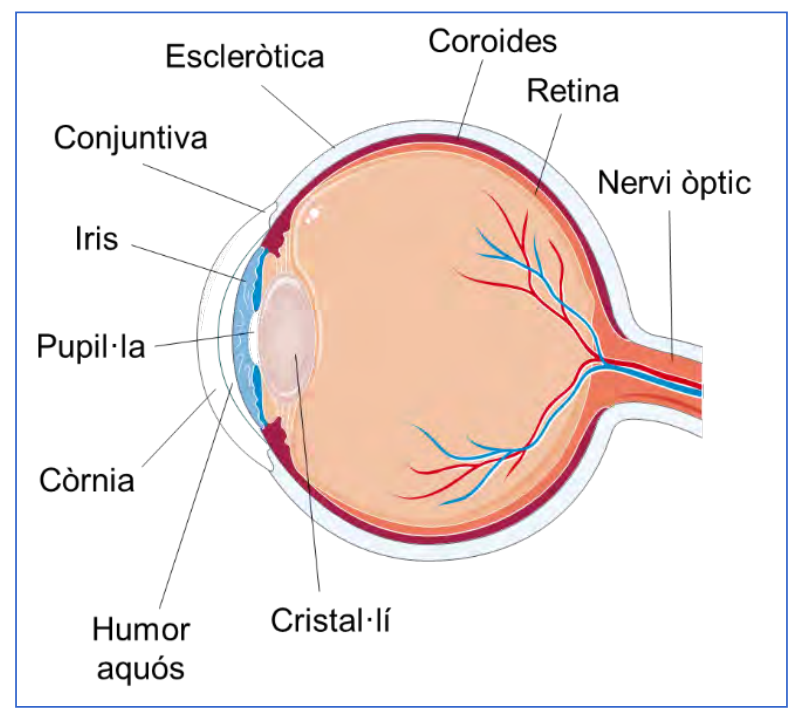

Figura 1. Estructura de l'ull humà (basat en Brill Pharma).

En la part anterior de l'ull es troba la còrnia, que és la part transparent de la capa protectora de l'ull que permet l'entrada de la llum. L'iris és la part acolorida de l'ull que es contrau i es dilata, i així regula la quantitat de llum que entra a l'ull. La llum travessa la pupilla fins al cristalllí, que enfoca la llum a la retina (part posterior de l'ull). Les fibres nervioses de la retina transporten la informació al cervell a través del nervi òptic, que és on es formarà la imatge.

El segment anterior de l'ull està ple d'un líquid transparent anomenat humor aquós produït pel cos ciliar. Aquest líquid surt de l'ull a través de la pupilla per ser absorbit cap al torrent sanguini mitjançant el sistema de drenatge de l'ull (una malla de canals de drenatge que es troba al voltant de la vora externa de l'iris). Un drenatge adequat ajuda a mantenir la pressió ocular en un nivell normal. La producció, el flux i el drenatge d'aquest líquid és un procés actiu i continu necessari per a la salut de l'ull. La pressió interna de l'ull (pressió intraocular) depèn de la quantitat de líquid que hi hagi a l'ull. Si el sistema de drenatge funciona apropiadament, el líquid pot drenar-se lliurement sense acumular-se (Portillo, 2001).

\subsubsection{Glaucoma}

El glaucoma és una malaltia neurodegenerativa comuna que afecta les cèlllules del ganglió de la retina (RGCs) i els seus axons. És la segona causa de ceguera crònica i la principal causa de ceguera irreversible a escala mundial (Tham et al., 2014). L'etiologia del glaucoma és desconeguda, però s'han identificat diversos factors de risc importants, incloent-hi la pressió intraocular elevada degut a un desequilibri entre la producció i la sortida de l'humor aquós (Henderer i Rapuano, 2006; Porth, 2007). 
El resultat final de la lesió del nervi òptic és la mort de les RGCs i, en la majoria dels casos, sembla que es produeix per vies d'autodestrucció apoptòtica (Fu et al., 2009). Els primers canvis en l'estructura dendrítica de les RGCs tenen conseqüències crítiques sobre l'eficàcia sinàptica i podrien ser la base dels dèficits funcionals en el glaucoma. Això es tradueix en una pèrdua del camp visual progressiva $a$ mesura que avança el curs de la malaltia. Per altra banda, les dones es veuen més afectades pel glaucoma per la seva major prevalença de glaucoma d'angle tancat, així com per la seva longevitat relativament més gran. Com que s'estima que les dones presenten el doble de deficiència visual i ceguera en general, en comparació amb els homes, s'hauria de prestar especial atenció al lliurament de serveis d'atenció ocular a les dones (Quigley i Broman, 2006).

En un ull sa, el fluid que surt cap a fora a través de l'angle de drenatge manté una pressió estable. Ara bé, si l'angle de drenatge es troba bloquejat, el fluid no podrà sortir de l'ull, de manera que s'incrementarà la pressió intraocular. S'ha trobat que hi ha dues categories principals de glaucoma: el glaucoma d'angle obert i el glaucoma d'angle tancat (figura 2). El terme «angle» fa referència a l'angle de drenatge intern de l'ull que controla la sortida del fluid produït contínuament dins de l'ull. Si el fluid aconsegueix accedir a l'angle de drenatge, estarem davant d'un glaucoma d'angle obert; en canvi, si l'angle de drenatge es troba bloquejat i el fluid no aconsegueix arribar-hi, estarem davant d'un glaucoma d'angle tancat (Boyd, 2019).

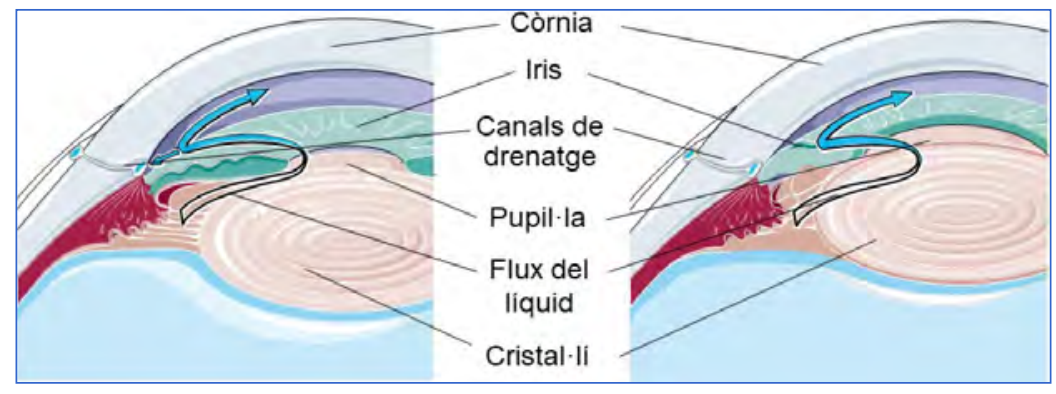

Figura 2. Glaucoma d'angle obert (esquerra) i glaucoma d'angle tancat (dreta) (basat en Glaucoma Research Foundation).

\subsubsection{Glaucoma d'angle obert}

Aquest tipus de glaucoma no presenta signes d'advertència ni símptomes obvis durant les primeres etapes. A mesura que la malaltia progressa, es desenvolupen punts cecs en la visió perifèrica lateral. La majoria de persones amb glaucoma d'angle obert no noten cap canvi en la seva visió fins que el dany és bastant greu.

\subsubsection{Glaucoma d'angle tancat}

Les persones amb risc de desenvolupar glaucoma d'angle tancat en general no mostren símptomes abans d'un atac. Alguns símptomes inicials d'un atac poden incloure: visió borrosa, halo de llum, mals de cap lleus o dolor en l'ull.

Un atac de glaucoma d'angle tancat inclou els següents símptomes:

- Dolor sever a l'ull o al front.

- Envermelliment de l'ull.

- Disminució de la visió o visió borrosa.

- Halo de llum.

- Mal de cap. 
- Nàusees i/o vòmits.

D'entre els factors de risc destaquem:

- $>40$ anys.

- Antecedents amb glaucoma.

- Ascendència africana, hispana o asiàtica.

- Pressió intraocular elevada.

- Hipermetropia o miopia.

- Lesions de l'ull prèvies.

- Ús perllongat d'esteroides.

- Tenir la còrnia prima al centre.

- Sofrir un estretament del nervi òptic.

- Patir diabetis, HTA, migranya, mala circulació de la sang i altres problemes de salut que afectin tot el cos.

Els objectius més recents del tractament del glaucoma són evitar el dany glaucomatós i el dany nerviós, i preservar el camp visual i la qualitat de vida total dels pacients, amb els mínims efectes adversos (Lora i Andr, 2005). Això requereix tècniques de diagnòstic apropiades i exàmens de seguiment, i una selecció judiciosa de tractaments per al pacient. Si bé la pressió intraocular és tan sols un dels principals factors de risc per al glaucoma, actualment la base del tractament del glaucoma consisteix a reduir-la a través de diversos fàrmacs i/o tècniques quirúrgiques. Alguns exemples de fàrmacs utilitzats en el glaucoma són (Lora i Andr, 2005; Bowling, 2016):

- Agonistes $\alpha$-adrenèrgics: disminueixen la producció d'humor aquós i n'augmenten el drenatge. Exemples: brimonidina, apraclonidina.

- Antagonistes $\beta$-adrenèrgics: disminueixen la producció d'humor aquós. Exemples: timolol, betaxolol.

- Inhibidors de l'anhidrasa carbònica: disminueixen la producció d'humor aquós. Exemples: dorzolamida, brinzolamida.

- Miòtics: augmenten el drenatge de l'humor aquós. Produeixen miosi i acomodació. Exemples: pilocarpina, carbacol.

- Anàlegs de la prostaglandina: augmenten el drenatge de l'humor aquós per via uveoescleral. Exemples: latanoprost, bimatoprost.

\subsubsection{Flavonoides}

El glaucoma és una malaltia neurodegenerativa multifactorial; per tant, una possible estratègia terapèutica seria l'ús de substàncies amb activitat neuroprotectora per tal de frenar la progressió de la malaltia. En aquest sentit, els flavonoides poden constituir un candidat adequat per al tractament del glaucoma.

El flavonoide d'origen natural té un baix pes molecular que pertany a la classe estructural 2-fenilbenzopiranona. En el seu estat pur, presenta estructures en forma d'agulles grogues. És pràcticament insoluble en aigua, moderadament soluble en alcohol calent i soluble en KOH i DMSO diluïts. També és incompatible amb agents oxidants forts (Corradini et al., 2010).

Durant segles s'han utilitzat els flavonoides d'origen natural amb fins medicinals. Per exemple, la flor de la passió (Passiflora caerulea), que conté nivells elevats de flavonoides, s'ha utilitzat de manera eficaç en el tractament de l'asma, l'insomni intransigent, la malaltia de Parkinson i la neuràlgia. Els flavonoides són un component important també de la camamilla (Matricaria chamomilla), que es reconeixen per les seves propietats antiespasmòdiques, antiinflamatòries, calmants, antibacterianes, antial-lèrgiques i antitu-morals (Hirano, Oka i Akiba, 1989; Cazarolli et al., 2008). 


\subsubsection{Nanopartícules}

L'ull és únic quant a la seva naturalesa anatòmica i fisiològica i els seus mecanismes de defensa. Això fa que l'orientació de medicaments cap als teixits oculars sigui un dels majors reptes en l'administració de medicaments. La instillació tòpica de medicaments a través de gotes per als ulls és la via d'administració més important i ben acceptada per al tractament de diversos trastorns oculars, com el glaucoma (Almeida et al., 2014).

Les formulacions farmacèutiques convencionals, com les solucions i suspensions, tenen molts inconvenients, exemplificats per l'eliminació precornial ràpida, el drenatge per gravetat, el recanvi normal de llàgrimes, la instilllació freqüent, el metabolisme enzimàtic, el drenatge nasolacrimal, l'absorció conjuntival i l'absència d'alliberació controlada i de propietats bioadhesives. El temps de residència de la majoria de solucions oculars convencionals és de 5 a 25 minuts, i tan sols s'absorbeix de l'1 al 10 \% del fàrmac aplicat tòpicament (Almeida et al., 2013; Taha et al., 2014). A més, una gran part del fàrmac absorbit pot arribar a la via sistèmica i produir efectes adversos sistèmics. La limitada permeabilitat de les membranes oculars contribueix a la baixa absorció de fàrmacs oculars, que implica una curta durada de l'efecte terapèutic i requereix un règim de dosificació freqüent.

Una possible estratègia per augmentar o perllongar el temps de contacte de les formulacions oftàlmiques amb els teixits oculars és utilitzar nanopartícules carregades amb el principi actiu.

Les nanopartícules es defineixen generalment com a partícules amb una dimensió compresa entre 1 i $1.000 \mathrm{~nm}$. Aquesta definició les situa en un domini similar al de les partícules ultrafines com un subconjunt de partícules col-loidals (Christian et al., 2008; Lungu et al., 2015). Un sistema col-loidal és en general un sistema de dues fases format per un medi dispersant i un medi dispers. El coneixe-ment de les propietats i característiques de les nanopartícules ha fet possible aplicar-les en diverses tecnologies en àrees com l'electrònica i la medicina, entre d'altres.

\subsubsection{Nanopartícules lipídiques}

Dins de les nanopartícules trobem les que són de naturalesa lipídica, que s'utilitzen com a sistemes d'alliberament de medicaments. Aquestes nanopartícules es poden obtenir a partir de lípids sòlids o una barreja de lípids sòlids i líquids, i s'estabilitzen amb emulsionants. Per tant, les nanopartícules formen part dels sistemes d'alliberament, és a dir, sistemes que transporten substàncies d'interès, com els fàrmacs.

Les nanopartícules lipídiques tenen molts avantatges en comparació amb altres sistemes de partícules, com per exemple:

- Facilitat de producció a gran escala.

- Biocompatibilitat i biodegradabilitat dels materials.

- Baix potencial de toxicitat.

- Possibilitat d'alliberament controlat i modificat dels medicaments.

- Millor solubilitat farmacològica.

- Possibilitat d'incorporar fàrmacs tant hidròfils com lipòfils.

Els paràmetres més importants en la caracterització són: la mida de les nanopartícules, la polidispersió, el potencial Z, el polimorfisme, el grau de cristallització, la càrrega de fàrmacs, l'eficiència d'encapsulació (EE) i l'alliberació de fàrmacs (Magalhães et al., 2020). En general podríem dir que, entre les raons més importants d'utilitzar les nanopartícules lipídiques com a alternativa a les nanopartícules polimèriques, hi trobem la facilitat de producció a gran escala i el seu baix potencial de toxicitat.

La primera generació de transportadors basats en lípids van ser les nanopartícules sòlides lipídiques (SLN), que es formulen, com el seu nom indica, a partir de lípids sòlids a temperatura 
corporal i estabilitzats amb emulsionants. Les SLN tenen nombrosos avantatges, entre els quals destacaríem:

- Protecció dels fàrmacs enfront de condicions ambientals desfavorables.

- Facilitat de la producció a gran escala mitjançant tècniques

- d'homogeneïtzació d'alta pressió.

- Biocompatibilitat.

- Biodegradabilitat.

Ara bé, també hi podem trobar alguns inconvenients:

- Baixa eficiència de càrrega de fàrmacs degut a la seva estructura cristal-lina perfecta.

- Possibilitat d'expulsió dels fàrmacs degut al procés de cristal-lització durant les condicions d'emmagatzematge.

- Alliberament d'esclat inicial.

En les SLN els fàrmacs s'orienten entre les cadenes d'àcids grassos, i durant els períodes d'emmagatzematge i durant els canvis polimòrfics hi ha una tendència a l'expulsió del fàrmac dissolt prèviament en les NP.

\subsubsection{Transportadors lipídics nanoestructurats}

Per tal de superar les limitacions de les SLN es va dissenyar una nova generació de nanopartícules lipídiques que són els transportadors lipídics nanoestructurats (NLC), formats a partir de la barreja de lípids sòlids i líquids, amb una matriu sòlida amorfa (figura 3). La matriu resultant dels NLC mostra una disminució del punt de fusió en comparació amb el lípid sòlid original, però la matriu continua sent sòlida a temperatura corporal (Garzón et al., 2008). Segons la forma de producció i la composició de la barreja de lípids, s'obtenen diferents tipus d'NLC.

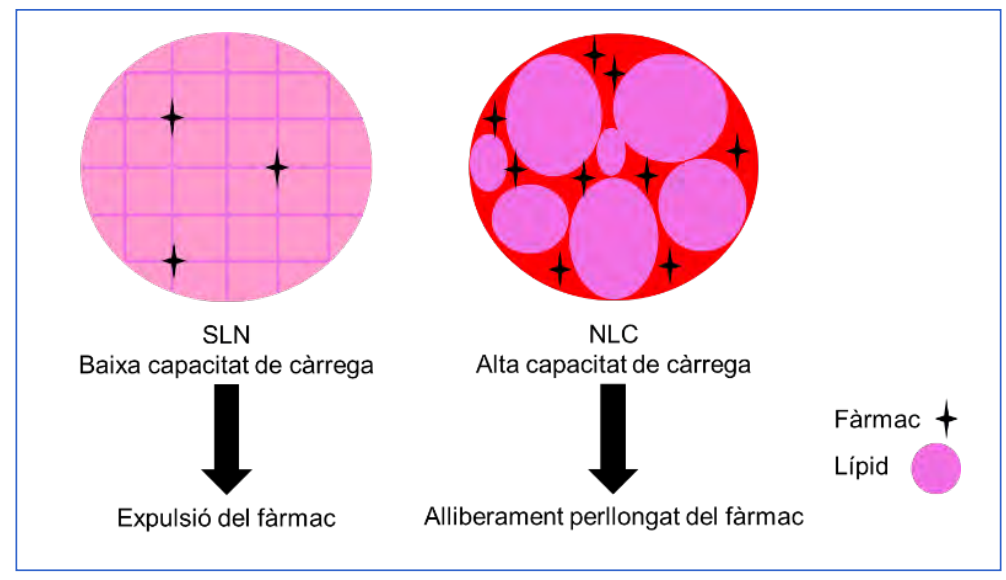

Figura 3. Esquema de les SLN i els NLC que mostra la ubicació del fàrmac dins la matriu lipídica (basat en Ghasemiyeh i Mohammadi-Samani, 2018). 
TAULA 1. AVANTATGES DELS NLC RESPECTE LES SLN I INCONVENIENTS

DELS NLC (MÜLLER, RADTKE I WISSING, 2002; TRUJILLO I WRIGHT, 2010).

\begin{tabular}{|c|c|}
\hline Avantatges & Inconvenients \\
\hline $\begin{array}{l}\text { Increment de la capacitat de càrrega de } \\
\text { fàrmacs. }\end{array}$ & Pocs estudis toxicològics sobre la retina. \\
\hline $\begin{array}{c}\text { Eviten l'expulsió del fàrmac en condicions } \\
\text { de producció i emmagatzematge. }\end{array}$ & $\begin{array}{c}\text { La mida de les partícules, la càrrega, el temps } \\
\text { d'exposició i la concentració de fàrmacs són } \\
\text { també factors importants en la toxicitat de la } \\
\text { retina. }\end{array}$ \\
\hline Alliberament perllongat del fàrmac. & $\begin{array}{l}\text { La majoria d'estudis només van ser d'avaluació } \\
\text { in vitro. }\end{array}$ \\
\hline \multicolumn{2}{|l|}{ Protecció del fàrmac. } \\
\hline Estabilitat fisicoquímica a llarg termini. & \\
\hline
\end{tabular}

\subsection{Mètodes experimentals}

\subsubsection{Materials}

Com s'ha comentat anteriorment, els NLC estan formats per una barreja de lípids sòlids i líquids. En el present treball es van seleccionar dos lípids químicament diferents, el Precirol ${ }^{\circledR}$ ATO 5 com a lípid sòlid i un oli essencial d'origen natural com a lípid líquid, perquè tenen bona miscibilitat a la tempe-ratura desitjada i amb el principi actiu, són estables, no són irritants i no presenten toxicitat. Es va escollir el Tween ${ }^{\circledR} 80$ com a agent humectant.

El Precirol ${ }^{\circledR}$ ATO 5 és una barreja de mono-, di- i triglicèrids de l'àcid palmític (C16) i l'àcid esteàric (C18). Es tracta d'una pols de color blanc amb un punt de fusió entre $50^{\circ} \mathrm{C}$ i $60{ }^{\circ} \mathrm{C}($ Gattefossé Pharmaceuticals, 2020) que és compatible i miscible amb el flavonoide i l'oli d'origen natural. És un lípid d'ampli ús com a agent modificador de l'alliberació de fàrmacs i formador de matrius lipídiques (Bonillo Martínez, Margarit Bellver i Rodríguez Galán, 2017).

L'oli d'origen natural destaca per les seves propietats nutritives. Pot ser aprofitat, per exemple, pel seu alt contingut en àcids grassos poliinsaturats, tals com el linoleic i el linolènic, indispensables per a la regeneració de cèllules noves, o com per exemple per mantenir la frescor de la pell, combatre infeccions i tractar diferents malalties (Raut i Karuppayil et al., 2014). En el nostre cas no està aprovat per les agències reguladores per a la via ocular. Caldrà fer més estudis per demostrar si és apte per formar part de diverses formes farmacèutiques.

Per altra banda, trobem el Tween ${ }^{\circledR} 80$, conegut també com polisorbat 80 , que és un humectant que facilita l'estabilització de la formulació d'NLC. És un líquid groc viscós que no és irritant per a pells i mucoses. Per altra banda, s'ha vist que incrementa la permeabilitat de la formulació a través dels vasos (Imperiale et al., 2018).

\subsubsection{Preparació dels transportadors lipídics nanoestructurats}

Per a la preparació dels NLC es va fer servir el mètode d'homogeneïtzació a alta pressió en calent (figura 4) d'acord amb Souto et al. (2005), que és el més utilitzat per a l'obtenció dels NLC, pel fet que pot ser fàcilment escalat a nivell industrial. En aquest mètode s'apliquen elevades quantitats d'energia que generen una força de tall important, una turbulència i una cavitació al material oliós, i poden processar dispersions de fins al $40 \%$ de lípids, cosa que dona lloc a NLC amb baix IP (Jenning, Thüne- 
Edusfarm 13 (2021), 1-16

ISSN: $1886-6271$

mann i Gohla, 2000). En general s'afavoreix la formació de partícules fines incrementant la temperatura, la pressió i/o el nombre de cicles d'homogeneïtzació.

Es van preparar per separat les dues fases constituents de la formulació: la fase aquosa amb el tensioactiu Tween ${ }^{\circledR} 80$ i aigua MilliQ, i la fase oliosa amb el lípid sòlid (Precirol ${ }^{\circledR}$ ATO 5) i el lípid líquid (oli d'origen natural). Les dues fases es van escalfar fins als $80{ }^{\circ} \mathrm{C}$, temperatura en la qual els dos lípids es fonen i formen una única fase. A continuació es va dispersar la fase aquosa sobre l'oliosa i es va agitar a alta velocitat amb l'Ultraturrax T10 Basic per formar una preemulsió que des-prés es va passar a l'homogeneïtzador d'alta pressió FPG 12800, amb les condicions de producció de $80{ }^{\circ} \mathrm{C}$. L'homogeneïtzador es va engegar 30 minuts abans de començar la preparació per permetre la recirculació d'aigua MilliQ calenta a $80{ }^{\circ} \mathrm{C}$. Finalment, la nanoemulsió obtinguda es va refredar fins a temperatura ambient, recristal-litzant el lípid i formant els NLC.

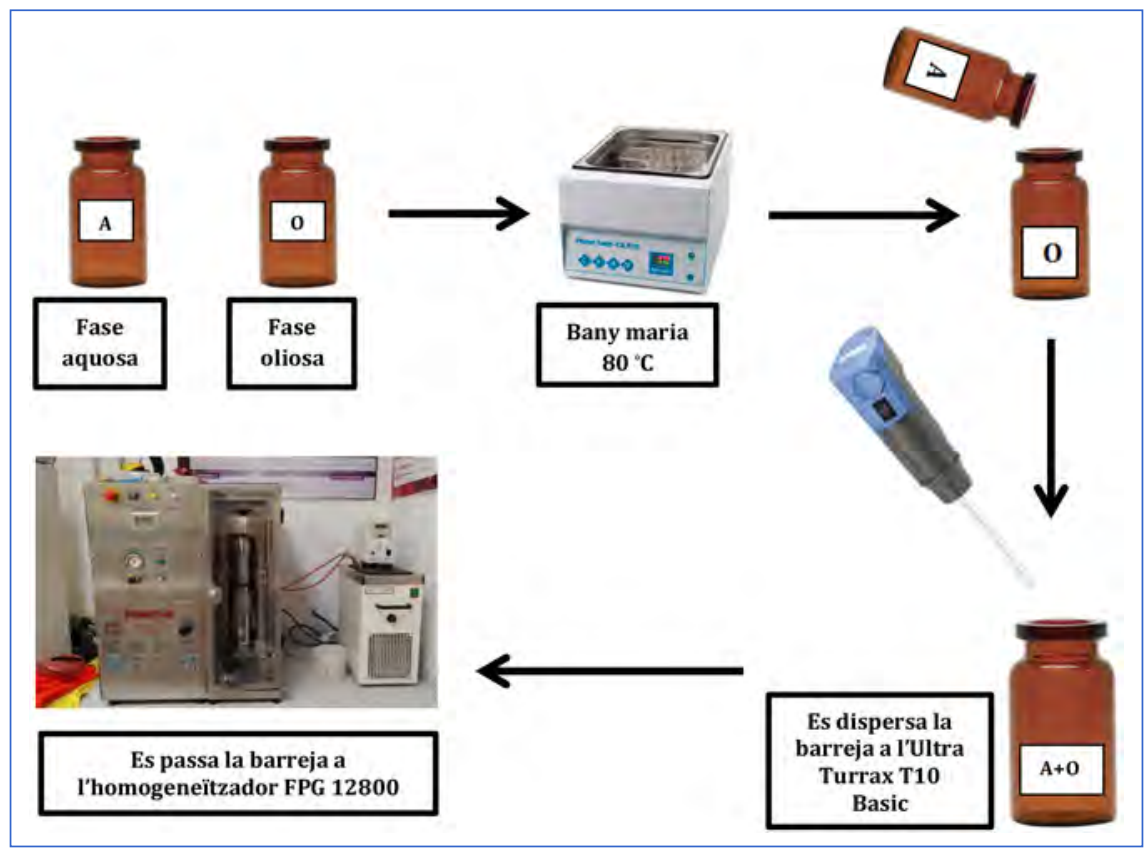

Figura 4. Esquema del mètode d'homogeneïtzació a alta pressió en calent dels NLC. Fase aquosa: $\mathrm{H}_{2} \mathrm{O}$ MilliQ + Tween $^{\circledR} 80$; fase oliosa: Precirol ${ }^{\circledR}$ ATO 5 + oli essencial d'origen natural.

\subsubsection{Caracterització dels transportadors lipídics nanoestructurats}

\subsubsection{Mida i índex de polidispersió}

Un cop preparats els NLC es va esperar 24 hores abans de dur a terme la caracterització fisicoquímica, per assegurar que la nanopartícula finalitzés el procés de solidificació, de manera que totes les nanopartícules estiguessin correctament formades.

La mida mitjana i l'IP dels NLC es van determinar mitjançant espectroscòpia de correlació fotònica amb el Zetasizer Nano ZS a temperatura ambient, utilitzant cubetes de poliestirè d'un sol ús després d'una dilució d'1:10 NLC amb aigua MilliQ. 


\subsubsection{Eficiència d'encapsulació}

L'eficiència d'encapsulació (EE) es defineix com el percentatge de massa del fàrmac contingut en els NLC en relació amb la massa total del fàrmac del sistema (dins i fora dels NLC) (Sponton, 2016; Suárez i Brito, 2020). La mesura de l'EE es va realitzar amb un cromatògraf líquid d'alta eficàcia (high performance liquid chromatography, HPLC) Waters, compost per una bomba Waters 600 Controller, l'injector Waters 717 Plus Autosampler i un detector Waters 2996 Photodiode Array Detector.

- Fase mòbil:

- $\mathbf{A} \rightarrow$ Àcid acètic $2 \%$.

- B $\rightarrow$ Metanol.

- Columna: Kromasil 100-5-C18 d'un diàmetre de 4,6 x 150 mm.

- Longitud d'ona $(\lambda): 300 \mathrm{~nm}$.

- Flux isocràtic: $0,9 \mathrm{~mL} / \mathrm{min}$.

L'EE es calcula amb l'equació 1 :

$$
\mathrm{EE}(\%)=\frac{\mathrm{C}_{\text {total }}-\mathrm{C}_{\text {lliure }}}{\mathrm{C}_{\text {total }}} \cdot 100 \quad \text { (Eq. 1) }
$$

\subsubsection{Disseny factorial central dels transportadors lipídics nanoestructurats}

Per tal d'optimitzar la formulació dels NLC amb un mínim nombre d'experiments es va seleccionar un disseny factorial central de $3^{3}$ per estudiar l'efecte de 3 variables independents: nombre de cicles, temps d'Ultraturrax i pressió d'homogeneïtzació, en la mida mitjana (mesurada per espectroscòpia de correlació fotònica) i en l'IP dels NLC (Sankalia et al., 2004; calcium chloride concentration and hardening time on \% entrapment, time required for 50\% (T50 Colombo, 2017).

L'anàlisi de les dades per a la selecció de la fórmula optimitzada es va realitzar mitjançant el software Statgraphics XVIII.

\subsubsection{Estudi de tolerància ocular: test HET-CAM}

El test HET-CAM és una alternativa al test de Draize ocular in vivo per a la detecció de productes potencialment irritants en la mucosa ocular (MB Research Labs, 2019). S'ha demostrat que aquest test és un mètode qualitatiu per avaluar la irritabilitat potencial dels productes químics. La membrana corioalllantoide (CAM) és un teixit complet que conté artèries, venes i capilllars, i és tècnicament fàcil d'estudiar. Respon davant de les lesions amb un procés inflamatori similar al que s'observaria al teixit conjuntival dels ulls dels conills. La seva vascularització ben desenvolupada proporciona un model ideal per a estudis d'irritació ocular (Sánchez López, 2018). Els compostos per assajar es posen en contacte amb la membrana corioal-lantoide de l'ou de gallina. L'aparició de les lesions vasculars o de coagulació en resposta a un compost és la base per emprar aquesta tècnica com a indicació de probabilitat que el producte danyi les membranes mucoses (especialment l'ull) in vivo.

Per realitzar l'assaig es van utilitzar ous de gallina embrionats de 10 dies. Es retira acuradament la closca de l'ou i s'elimina la membrana testàcia. A continuació s'humiteja la membrana amb una solució de $\mathrm{NaCl}$ al 0,9 \% a temperatura ambient sense danyar cap vas sanguini subjacent amb el fòrceps. Després es retira l'excés de $\mathrm{NaCl}$. Seguidament es dosifica $300 \mu \mathrm{L}$ de mostra o controls i s'observa contínuament durant 5 minuts per si hi ha aparició d'hemorràgia, lisi i/o coagulació. 
El potencial irritant ocular es pot calcular mitjançant l'equació 2:

$$
\mathrm{IIO}=\frac{(301-H) \cdot 5}{300}+\frac{(301-V) \cdot 7}{300}+\frac{(301-C) \cdot 9}{300} \quad \text { (Eq. 2) }
$$

$\mathbf{H}$ és el temps (en segons) per a l'aparició de l'hemorràgia, $\mathbf{V}$ és el temps (en segons) per a l'aparició de la vasoconstricció i C el temps (en segons) per a l'aparició de la coagulació. Finalment es classifica la formulació d'acord amb l'índex d'irritació ocular (IIO) (taula 2).

TAULA 2. CLASSIFICACIÓ DELS PRODUCTES D’ACORD AMB L'IIO.

\begin{tabular}{|c|c|}
\hline IIO & CLASSIFICACIÓ \\
\hline $0-0,9$ & Pràcticament NO irritant \\
\hline $1-4,9$ & Lleugerament irritant \\
\hline $5-8,9$ & Moderadament irritant \\
\hline $9-21$ & Irritant \\
\hline
\end{tabular}

En considerar la «Reducció, Refinament i Reemplaçament» de l’experimentació amb animals, és molt important reconèixer que la prova d'ous embrionats és un cas límit entre sistemes in vivo i in vitro, i no entra en conflicte amb aspectes ètics i legals, especialment les lleis de protecció animal (Kishore et al., 2008) the potential irritancy of compounds was evaluated by observing adverse chan- ges that occur in chorioallantoic membrane CAM.

\section{Resultats}

A partir de la metodologia descrita al bloc 2.2 s'obtenen formulacions de transportadors lipídics nanoestructurats carregats de flavonoide amb un valor de la mida mitjana inferior a $200 \mathrm{~nm}$, un índex de polidispersió inferior a 0,2 i una eficiència d'encapsulació al voltant del $99 \%$.

Al test HET-CAM es va assajar el flavonoide per si mateix i els NLC carregats amb el flavonoide, en ous de gallina embrionats de 10 dies amb un pes comprès entre $50 \mathrm{~g} \mathrm{i} 60 \mathrm{~g}$ (Katiyar et al., 2014).

A la figura 5 es mostra el control negatiu i els controls positius de l'assaig, que es basen en els mateixos compostos que els emprats en l'estudi realitzat per l'equip d'Alvarado et al. (2015). El control negatiu representa una solució fisiològica formada per $\mathrm{NaCl}$ al 0,9 \% i s'observa clarament que no apareix cap signe d'hemorràgia, vasoconstricció i/o coagulació (figura 5, A i D). Com a controls posi-tius es van assajar dues substàncies: una amb propietats irritants a nivell ocular amb un efecte ràpid $(\mathrm{NaOH}$, figura $5, \mathrm{E}) \mathrm{i}$ una altra amb propietats irritants a nivell ocular però amb un efecte més lent (lauril sulfat sòdic, LSS, figura 5, F). En ambdós controls s'observen signes d'irritació ocular, però a temps diferents. 


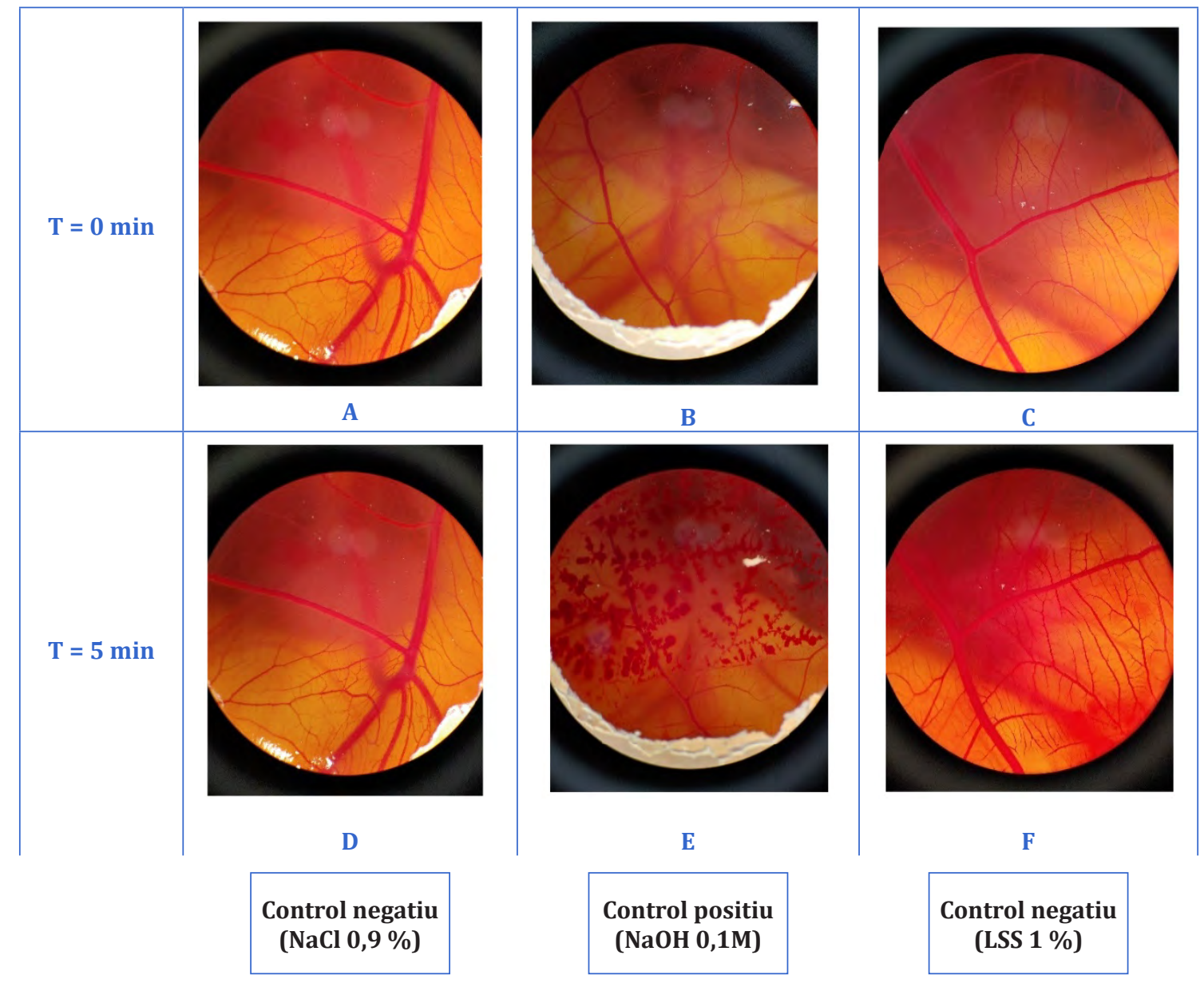

Figura 5. Controls de l'assaig HET-CAM.

A la figura 6 es mostren les imatges de les membranes corioal-lantoides després d'assajar: NLC amb flavonoide (figura 6, A i C) i flavonoide sol (figura 6, B i D ). Es pot observar que en cap de les proves les substàncies provoquen signes d'hemorràgia, vasoconstricció i coagulació. 


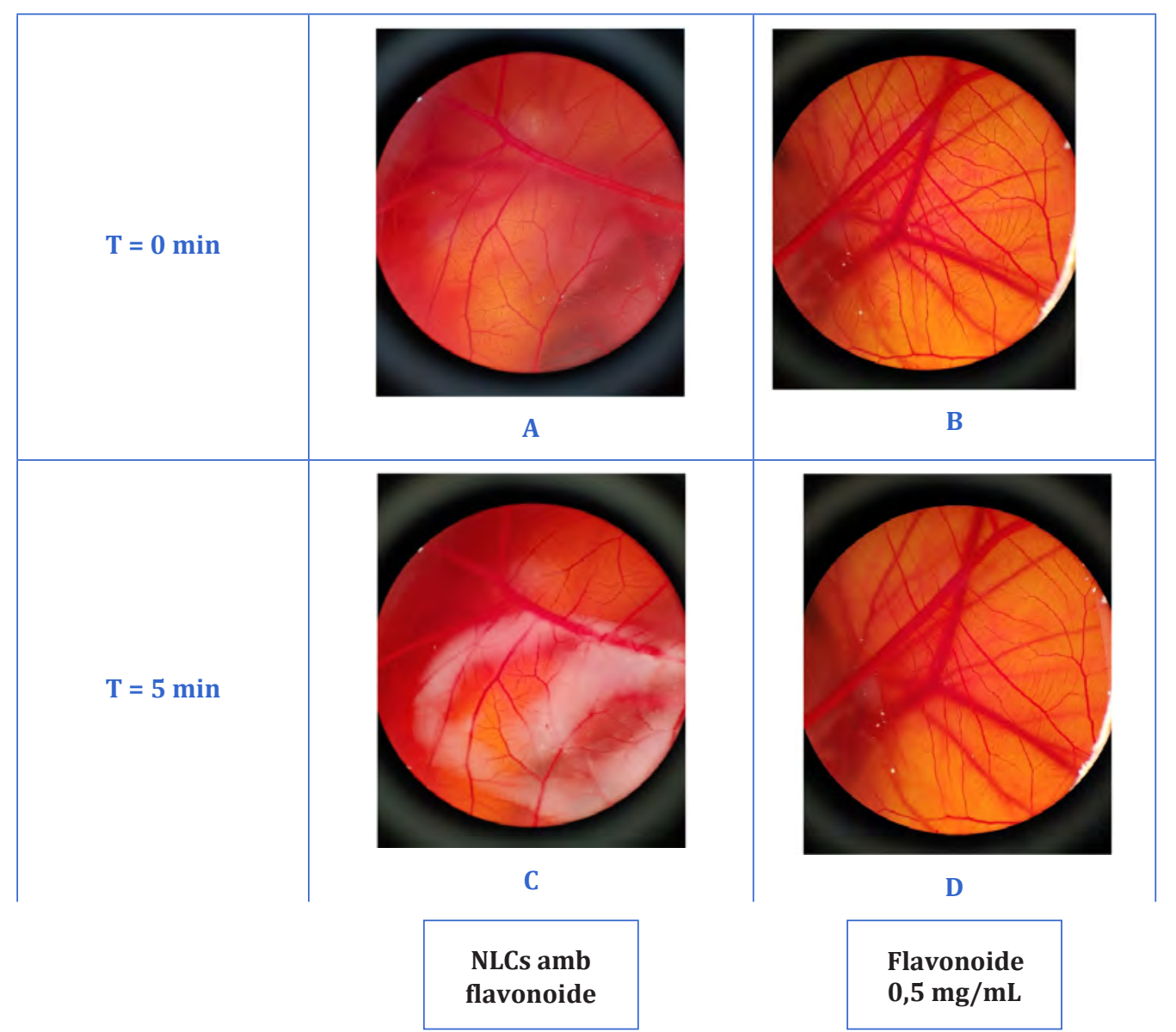

Figura 6. Imatges de l'assaig HET-CAM de les formulacions amb i sense flavonoide, i flavonoide sol.

Després d'obtenir els resultats representats a la taula 3, es va aplicar l'equació 2 per calcular l'índvex d'irritació ocular (IIO) a partir dels temps d'aparició d'hemorràgia, vasoconstricció i coagula-ció, i d'aquesta manera es va classificar cada substància. Es pot observar que al control negatiu es van enregistrar valors de $300 \mathrm{~s}$ (equivalents a $5 \mathrm{~min}$ ) degut al fet que no es van observar els signes. En canvi, als controls positius es van anotar els temps als quals es van observar els signes. En el cas del $\mathrm{NaOH} 0,1 \mathrm{M}$ es van observar ràpidament l'aparició de tots els signes, però l'LSS $1 \%$ va mostrar uns signes més tardans. Ambdós són classificats com a substàncies irritants segons l'IIO (taula 2).

Observant els resultats de les mostres d'NLC amb flavonoide no es va apreciar cap dels signes, fet que ens demostra que aquests NLC són bons candidats per al seu ús per via ocular. Ara bé, el flavonoide sol mostra un caràcter moderadament irritant, ja que s'ha observat una vasoconstricció al cap de pocs segons. Per tant, podríem afirmar que aquesta formulació (NLC + flavonoide) seria un bon candidat per al seu ús per via ocular en forma de gotes oftàlmiques, pel fet de ser pràcticament no irritant. Tot i això, caldria fer més estudis per acabar de demostrar aquesta característica i que les agències reguladores ens donessin el vistiplau. 


\section{TAULA 3. RESULTATS DEL TEST HET-CAM I CLASSIFICACIÓ DELS CONTROLS I DE LES MOSTRES.}

\begin{tabular}{|c|c|c|c|c|c|c|}
\hline Substància & Pes (g) & $\begin{array}{c}\text { Temps } \\
\text { H } \\
\text { (s) }\end{array}$ & $\begin{array}{c}\text { Temps } \\
\text { V } \\
\text { (s) }\end{array}$ & $\begin{array}{c}\text { Temps } \\
\text { C } \\
\text { (s) }\end{array}$ & $\mathrm{IIO} \pm \mathrm{DS}$ & Classificació \\
\hline $\mathrm{NaCl}(0,9 \%)$ & 57,60 & 300 & 300 & 300 & $0,07 \pm 0,00$ & $\begin{array}{l}\text { Pràcticament } \\
\text { no irritant }\end{array}$ \\
\hline $\begin{array}{c}\mathrm{NaOH} \\
(0,1 \mathrm{M})\end{array}$ & 54,69 & 18 & 40 & 26 & $19,06 \pm 0,00$ & Irritant \\
\hline LSS (1 \%) & 54,43 & 300 & 208,8 & 13 & $10,81 \pm 0,00$ & Irritant \\
\hline $\begin{array}{c}\text { NLC } \\
+ \\
\text { Flavonoide } \\
(0,5 \mathrm{mg} / \mathrm{mL})\end{array}$ & 55,92 & 300 & 300 & 300 & $0,07 \pm 0,00$ & $\begin{array}{l}\text { Pràcticament } \\
\text { no irritant }\end{array}$ \\
\hline $\begin{array}{l}\text { Flavonoide } \\
(0,5 \mathrm{mg} / \mathrm{mL})\end{array}$ & 56,25 & 300 & 15 & 300 & $6,72 \pm 0,00$ & $\begin{array}{c}\text { Moderadament } \\
\text { irritant }\end{array}$ \\
\hline
\end{tabular}

\section{Conclusions}

Com a resultat de la investigació realitzada s'han extret les següents conclusions:

- Es pot veure com la nanotecnologia pot aplicar-se al camp de l'oftalmologia amb el fi de millorar la qualitat de vida dels pacients amb glaucoma.

- S'ha comprovat que les nanopartícules lipídiques de segona generació proporcionen més avantatges que les de primera generació pel fet que aquestes presenten una sèrie de limitacions.

- A partir del disseny factorial central s'han aconseguit les millors condicions per obtenir una mida mitjana de partícula i un índex de polidispersió inferiors a $200 \mathrm{~nm}$ i 0,2, respectivament.

- Amb el test HET-CAM s'han pogut classificar els NLC carregats amb flavonoide com a pràcticament no irritants a nivell ocular a priori. Tot i que el flavonoide sol sí que produeix vasoconstricció, pel fet d'estar encapsulada no suposa cap problema.

- Calen fer més estudis a posteriori per demostrar que no és irritant a nivell ocular i per provar-ne l'eficàcia farmacològica.

La possibilitat de desenvolupar un producte comercialitzat basat en NLC per a una instillació diària de medicaments continua oberta per augmentar el compliment de la presa de la medicació per part del pacient.

\section{Bibliografia}

AlmeidA, H. et al. (2013) «Applications of poloxamers in ophthalmic pharmaceutical formulations: an overview».Expertopinionondrugdelivery,10(9),p.1223-1237.doi:10.1517/17425247.2013.796360.

AlmeidA, H. et al. (2014) «In situ gelling systems: A strategy to improve the bioavailability of ophthalmic phar maceutical formulations». Drug Discovery Today, 19(4), pp. 400-412. doi: 10.1016/j.drudis.2013.10.001.

Alvarado, H. L. et al. (2015) «Design and optimization of oleanolic/ursolic acid-loaded nanoplatforms for ocular anti-inflammatory applications». Nanomedicine: Nanotechnology, Biology, and Medicine. Elsevier Inc., 11(3), pp. 521-530. doi: 10.1016/j.nano.2015.01.004. 
Bonillo Martínez, A. D., Margarit Bellver, M. V. i Rodríguez Galán, I. C. (2017) «Matrix tablets based on amino acid-derived polyesteramide containing release modifiers». Journal of Drug Delivery Sci ence and Technology, 42, pp. 307-314. doi: 10.1016/j.jddst.2017.05.003.

Bowling, B. (2016) Oftalmología clínica. 8a ed. Elsevier.

Boyd, K. (2019) American Academy of Ophthalmology, Glaucoma. Disponible a: https://www.aao.org/saludocular/enfermedades/glaucoma-sintomas (accedit: 26 març 2020).

CAZARolli, L. H., et al (2008) Flavonoids: Prospective drug candidates, Mini-Reviews in Medicinal Chemistry, 8(13), pp. 1429-1440. doi: 10.2174/138955708786369564.

Christian, P. et al. (2008) «Nanoparticles: Structure, properties, preparation and behaviour in environmental media». Ecotoxicology, 17(5), pp. 326-343. doi: 10.1007/s10646-008-0213-1.

CоLомво, Е. (2017) Diseño de síntesis para manufactura reproducible de nanopartículas superparamagnéticas orientadas a aplicaciones biomédicas. Universidad Nacional del Litoral.

CORRADINI, E., et al. (2010) «Flavonoids: chemical properties and analytical methodologies of identification and quantitation in foods and plants», Natural Product Research, 25(5), pp. 469-495.

Fu, Q. L. et al. (2009) «Synaptic degeneration of retinal ganglion cells in a rat ocular hypertension glaucoma model». Cellular and Molecular Neurobiology, 29(4), pp. 575-581. doi: 10.1007/s10571-0099349-7.

GARZón S. et al. (2008) «Preparación de nanopartículas sólidas lipídicas (SLN), y de acarreadores lipídicos nanoestructurados (NLC)». Revista Mexicana de Ciencias Farmaceuticas, 39(4), pp. 50-66.

Gattefossé Pharmaceuticals (2020) Precirol ATO 5. Disponible a: https://www.gattefosse.com/precirol-ato-5 (accedit: 26 març 2020).

GHASEMiYeh, P. i Mohammadi-SAMAni, S. (2018) «Solid lipid nanoparticles and nanostructured lipid carriers as novel drug delivery systems: applications, advantages and disadvantages». Research in Pharmaceutical Sciences, 13(4), pp. 288-303. doi: 10.4103/1735-5362.235156.

GILLERon, L. et al. (1996) «Evaluation of a modified HET-CAM assay as a screening test for eye Toxicology in Vitro, 10(4), pp. 431-446. doi: 10.1016/0887-2333(96)00021-5.

GuPTA, H. et al. (2010) «Sparfloxacin-loaded PLGA nanoparticles for sustained ocular drug delivery». Nanomedicine: Nanotechnology, Biology, and Medicine. Elsevier Inc., 6(2), pp. 324-333. doi: 10.1016/ j.nano.2009.10.004.

HAGINo, S. et al. (1999) «Interlaboratory validation of in vitro eye irritation tests for cosmetic ingredients. (2) Chorioallantoic membrane (CAM) test». Toxicology in Vitro, 13(1), pp. 99-113. doi: 10.1016/ S0887-2333(98)00065-4.

L. (eds.) Goodman \& Gilman: The Pharmacological Basis of Therapeutics. 11a ed. McGraw Hill. Disponible a: https://oncouasd.files.wordpress.com/2015/06/goodman-farmacologia.pdf (accedit: 6 abril 2020).

HirAno, T., OKA, K. i AKIBA, M. (1989) «Antiproliferative effects of synthetic and naturally occurring flavonoids on tumor cells of the human breast carcinoma cell line, ZR-75-1». Research Communications in Chemical Pathology and Pharmacology, 64(1), pp. 69-78.

Imperiale, J. C., AcostA, G. B. i Sosnik, A. (2018) «Polymer-based carriers for ophthalmic drug delivery». Journal of Controlled Release. Elsevier, 285(maig), pp. 106-141. doi: 10.1016/j.jconrel.2018.06.031.

Jenning, V., ThünEMAnN, A. F. i GohlA, S. H. (2000) «Characterisation of a novel solid lipid nanoparticle carrier system based on binary mixtures of liquid and solid lipids». International Journal of Pharmaceutics, 199(2), pp. 167-177. doi: 10.1016/S0378-5173(00)00378-1.

KATIYAR, S. et al. (2014) «In situ gelling dorzolamide loaded chitosan nanoparticles for the treatment of glaucoma». Carbohydrate Polymers. Elsevier Ltd., 102(1), pp. 117-124. doi: 10.1016/j.carbpol.2013. 10.079.

Kishore, A. S. et al. (2008) «Hen egg chorioallantoic membrane bioassay: An in vitro alternative to draize eye irritation test for pesticide screening». International Journal of Toxicology, 27(6), pp. 449-453. doi: 10.1080/10915810802656996.

LoRA, M. i ANDR, I. (2005) «Diagnóstico y tratamiento». Gastroenterología y Hepatología Continuada,4(4), pp. 161-165. doi: 10.1016/s1578-1550(05)75134-2. 
LUnGU, M. et al. (2015) «Nanoparticles' promises and risks: Characterization, manipulation, and potential hazards to humanity and the environment». Nanoparticles' Promises and Risks: Characterization, Manipulation, and Potential Hazards to Humanity and the Environment, (4), pp. 1-355. doi: 10.1007/9783-319-11728-7.

MAGALHÃEs, J. et al. (2020) «Optimization of rifapentine-loaded lipid nanoparticles using a Quality-by-De sign strategy». Pharmaceutics, 12(1), pp. 1-13. doi: 10.3390/pharmaceutics12010075.

MB Research Labs (2019) Hen's Egg Test Chorioallantoic Membrane (HET-CAM). Alternative Eye Irritation Testing. Disponible a: http://www.mbresearch.com/hetcam.htm (accedit: 10 abril 2020).

McAlinden, C. (2014) «Selective laser trabeculoplasty (SLT) vs other treatment modalities for glaucoma: systematic review». Eye, 28(3), pp. 249-258. doi: 10.1038/eye.2013.267.

MülLER, R. H., RADTKE, M. i Wissing, S. A. (2002) «Solid lipid nanoparticles (SLN) and nanostructured lipid carriers (NLC) in cosmetic and dermatological preparations». Advanced Drug Delivery Reviews, 54(supl.), pp. 131-155. doi: 10.1016/S0169-409X(02)00118-7.

Porth, C. (2007) Fisiopatología, Salud-Enfermedad: un enfoque conceptual. 7a ed. Madrid: Editorial Médica Panamericana S. A.

Portillo, C. del (2001) Mi deseo para el futuro es que nadie tenga que vivir con el miedo de perder la vista. Verdugo-Ga. A. Jackson. Glaucoma Research Foundation. Disponible a: http://envejecimiento.csic. es/documentos/documentos/grf-glaucoma-01.pdf (accedit: 29 març 2020).

Quigley, H. i Broman, A. T. (2006) «The number of people with glaucoma worldwide in 2010 and 2020». British Journal of Ophthalmology, 90(3), pp. 262-267. doi: 10.1136/bjo.2005.081224.

RAUT, J. S., KARUPPAYIL, S. M. (2014) 'A status review on the medicinal proeprties of essential oils', Industrial Crops and Products, 62, pp 250-264. doi: 10.1016/j.indcrop.2014.05.055

SÁnchez LóPEZ, E. (2019) Practice 3: Ocular Tolerance of Nanospheres (HET-CAM). Barcelona.

SANKALIA, M. G. et al. (2004) «Evaluation and simultaneous optimization of papain entrapped in crosslinked alginate beads using 33 factorial design and the desirability function». Ars Pharmaceutica, 45(3),pp. 253-279.

SONI, G. i YADAV, K. S. (2015) «Applications of nanoparticles in treatment and diagnosis of leukemia». Materials Science and Engineering C. Elsevier B. V., 47, pp. 156-164. doi: 10.1016/j.msec.2014.10.043.

Souto, E. B. et al. (2005) «Preparation and characterization of n-dodecyl-ferulate-loaded solid lipid nanoparticles (SLN)». International Journal of Pharmaceutics, 295(1-2), p. 261-268. doi: 10.1016/ j.ijpharm.2005.02.005.

SPONTon, O. E. (2016) Desarrollo de nanopartículas biopoliméricas para la encapsulación de ácidos grasos poliinsaturados. Tesis.

SuÁrez, M. i Brito, C. (2020) «Encapsulation efficiency and load capacity of anthocyanins of Vaccinium floribundim kunt in zein nanoparticle». Infoanalítica, 8(1), pp. 83-97. doi: 10.26807/ia.v8i1.9.

TAнA, E. I. et al. (2014) «Design of liposomal colloidal systems for ocular delivery of ciprofloxacin». Saudi Pharmaceutical Journal. King Saud University, 22(3), pp. 231-239. doi: 10.1016/j.jsps.2013.07.003.

THAM, Y. C. et al. (2014) «Global prevalence of glaucoma and projections of glaucoma burden through 2040: A systematic review and meta-analysis». Ophthalmology. Elsevier Inc, 121(11), pp. 2081-2090. doi: 10.1016/j.ophtha.2014.05.013.

Trujillo, C. C. i Wright, A. J. (2010) «Solid lipid nanoparticles: production, characterization and applications».Journal of the American Oil Chemists'Society, 87(7), pp. 165-196. doi:10.1039/9781847550842.

Wiggs, J. L. i PASQUALE, L. R. (2017) «Genetics of glaucoma». Human Molecular Genetics, 26(R1), pp. R21R27. doi: $10.1093 / \mathrm{hmg} / \mathrm{ddx} 184$.

YADAV, K. S., Rajpurohit, R. i SHARma, S. (2019) «Glaucoma: Current treatment and impact of advanced drug delivery systems». Life Sciences. Elsevier, 221, pp. 362-376. doi: 10.1016/j.lfs.2019.02.029. 